\title{
Coherent diffraction imaging of a progressively deformed nanocrystal
}

\author{
Marcus C. Newton* \\ Physics \& Astronomy, University of Southampton, SO17 1BJ, UK \\ Xiaowen Shi, Ulrich Wagner, and Christoph Rau \\ Diamond Light Source, Harwell Oxford Campus, Didcot OX11 ODE, UK
}

(Dated: April 17, 2019)

\begin{abstract}
Imaging ordered materials with coherent x-rays holds great potential to improve our understanding of phenomena in complex materials systems where emergent behaviour can arise due to coupling of spin, lattice and orbital degrees of freedom. Coherent diffractive imaging (CDI) is a lensless imaging technique for probing the structure of materials in three-dimensions. Central to the success of the CDI method is the inversion of propagated wavefield information to recover a quantitative image of the illuminated crystalline structure. Present challenges faced with existing approaches to image recovery are often due to non-uniqueness of wave propagated forms of the electron density information that can cause prohibitive stagnation of the reconstruction algorithm. Here we report on a major advancement in image recovery that is able to recover the three-dimensional image of a $492 \mathrm{~nm}$ gold single crystal undergoing progressive deformation to a highly strained condition without the use of a priori information. Our findings also demonstrate the significance of robust image recovery techniques for revealing high resolution topological structure.
\end{abstract}

\section{INTRODUCTION}

Coherent diffractive imaging (CDI) is a form of lensless microscopy that can permit high resolution imaging where the use of conventional optics is not feasible. [1-5] The Bragg coherent $x$-ray diffractive imaging (BCXDI) variant of CDI utilises the periodicity in crystalline materials to image nanoscale structure with a typical sensitivity below a single angstrom in both two and threedimensions. [6, 7] The largely non-destructive penetration of electromagnetic waves enables BCXDI to probe nanoscale materials and provide strain information at the surface and throughout the bulk. When combined with ultra-fast pulses of coherent $\mathrm{x}$-rays as provided by an x-ray free electron laser (XFEL), BCXDI is an enabling tool for the study of time-varying dynamic structural phenomena in nanoscale materials. [8, 9] BCXDI therefore holds great promise for understanding complex systems where the interplay between spin, lattice and orbital degrees of freedom give rise to emergent behaviour and multi-functional properties. The ability that BCXDI holds for directly imaging materials and quantitative mapping of defects and strain in threedimensions at the surface and in the bulk, can greatly increase our understanding of how new phases emerge and influence material properties.[7, 10-12]

BCXDI is performed experimentally by illuminating a sufficiently sized nanoscale crystal with a spatially coherent x-ray wavefield such that the Bragg reflected wave is collected in the far-field Fraunhofer limit. If the coherence lengths exceed the dimensions of the nanocrystal, a two-dimensional projection of the resulting diffraction pattern is recorded. If the nanocrystal object is greater in size than the illuminating field, util-

\footnotetext{
* M.C.Newton@soton.ac.uk
}

ising the extents of the wavefield and a series of spatially overlapping recordings is often sufficient to provide adequate sampling.[13-15] The third dimension is obtained by collating successive recordings of twodimensional projections that result from either conformal rotations of the nanocrystal that rotate the Ewald sphere through the diffraction pattern, or continuous tuning of the x-ray energy to expand the Ewald sphere through the diffraction pattern.[6, 16-20] Prior refinements to the experimental geometry ensure that the acquired three-dimensional image is oversampled relative to the Shannon-Nyquist sampling frequency (i.e. twice the highest spatial frequency of the visible diffraction pattern fringes). An image of the electron density map of the nanocrystal object is recovered by inversion of the acquired scattering intensity of the wavefield. With sufficient oversampling and appropriate constraints, iterative computational methods are able to reconstruct lost phase information from intensity measurements to recover the real-space image. [21-23]

Essential to the utility of BCXDI is the inversion of the diffracted wavefield from an isolated crystalline object. BCXDI measurements are however routinely performed on crystalline materials that are deformed from their ideal structure due to the presence of strain that can result from phenomena including dislocations, surface effects, phase transitions, density waves and chemical reactions. The presence of strain that results in a significant distortion away from a regular arrangement of atoms throughout the material can prohibit recovery of the real-space image as existing methods for inversion fall short without further a priori knowledge of the materials condition. As a result, recent efforts have focussed on providing a route to robust reconstruction to circumvent stagnation in existing iterative methods. [24, 25]

Here we demonstrate robust inversion in the absence of a priori information where existing methods fail to 

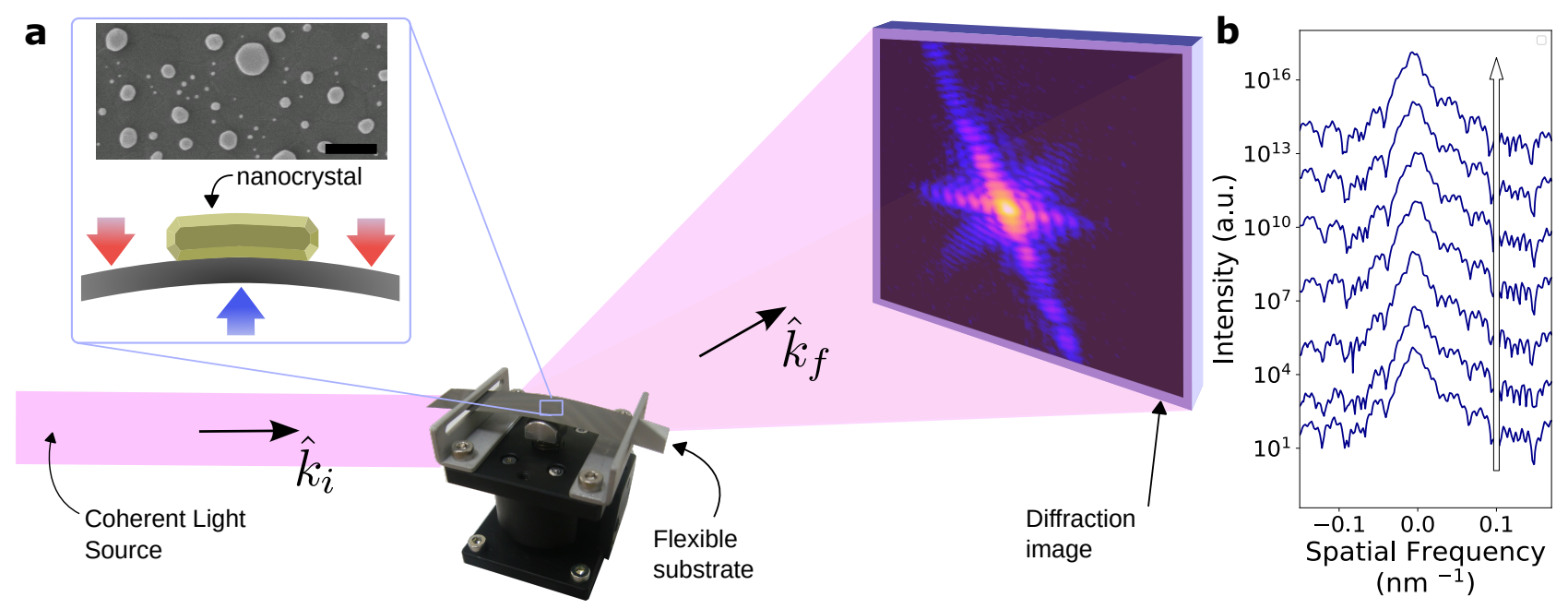

FIG. 1. Bragg Coherent X-ray Diffraction Imaging Experiment. (a) Experimental geometry showing mechanical deformation stage. Inset shows a schematic of the bending process and an SEM image of Au nanocrystals on a silicon surface (1 $\mu$ m scale bar). (b) Line scans of each diffraction pattern showing characteristic asymmetry in the fringe visibility which is attributed to the presence of strain in the nanocrystal.

recover three-dimensional images of a nanoscale crystal subject to controlled and progressively applied deformation. The method presented herein is of general application to CDI image recovery and was found to be particularly effective for highly strained nanoscale crystals.

Coherent diffractive imaging experiments record the scattering intensity field $I(\mathbf{q})$ in the form of an interference pattern that closely approximates the square modulus of the Fourier transform of the object function $\hat{\rho}(\mathbf{q})$ (See Appendix). Inversion is routinely accomplished using a discrete Fourier transform such as the Fast Fourier Transform (FFT) to recover the electron density map of the object $\rho(\mathbf{r})=|\rho(\mathbf{r})| e^{i \phi(\mathbf{r})}$. It was previously shown that Fourier amplitude inversion in one-dimension is susceptible to non-uniqueness while in higher dimensions, it is pathologically rare. $[14,26]$ If the object contains phase information beyond the range of $|\phi(\mathbf{r})| \leq \frac{\pi}{2}$, it is found empirically that such objects pose a greater challenge for phase retrieval and often contain spatially periodic amplitude gaps in the recovered electron density. This is understood as resulting from the equivalence in the Fourier transform modulus of all wavefield propagation distances between the real object and the detector plane where in general the propagated wave will acquire fringes with increasing phase information. [23] If the object contains phase information that occupies a range beyond $\pi$, there is an increased possibility that the object will resemble a propagated version of itself (that is spatially truncated).[26, 27] Standard projection algorithms might therefore stagnate on the propagated wavefield of the object where a greater number of similar solutions are likely to exist. In such cases, attempts to restrict the phase during inversion is unlikely to eliminate propagated wavefields from the recovered object. It is for this reason that a general route to image recovery of objects with phase spanning a range beyond $\pi$ is needed.

\section{EXPERIMENTAL METHODS}

\section{A. Reweighted two-dimensional phase retrieval}

Reweighted two-dimensional (2D) phase retrieval provides a more robust approach that is less prone to stagnation at a propagated wavefield of the object than standard gradient search methods as it does not seek a single minimum and explores the solution space to a broader extent. Its formulation is contrasted with that of the standard Hybrid Input-Output (HIO) algorithm.[23, 28, 29] Reciprocal-space $\varepsilon_{\mathrm{M}}^{2}$ and realspace $\varepsilon_{\mathrm{S}}^{2}$ error metrics are employed as with the HIO method that enforce (1) a reciprocal-space modulus constraint $\hat{\boldsymbol{P}}_{\mathrm{M}}$ operating on the object amplitude such that $|\hat{\rho}(\mathbf{q})|=\sqrt{ } I(\mathbf{q})$ and (2) a real-space support constraint operator $\boldsymbol{P}_{\mathrm{S}}$ which sets to zero a proportion of voxels that are outside of a given volume where the object is assumed to exist. The support constraint spatially restricts a proportion of equivalent solutions that are propagated away from the object and consist of a spatially broader amplitude distribution. The reciprocalspace modulus constraint was modified to include a weighting factor $W(\mathbf{r})$ that penalises voxels that vary significantly between iteration (See Appendix):

$$
\begin{aligned}
\varepsilon_{\mathrm{M}}^{2}(\rho) & =\left\|W^{(n)}(\mathbf{r})\left(\boldsymbol{P}_{\mathrm{M}} \rho(\mathbf{r})^{(n)}-\rho(\mathbf{r})^{(n)}\right)\right\|_{2}^{2} \\
W^{(n)}(\mathbf{r}) & =\left(\left|\rho(\mathbf{r})^{(n-1)}\right|+\epsilon_{0}\right)^{-1}
\end{aligned}
$$

where $\epsilon_{0} \ll\left|\rho(\mathbf{r})^{(n-1)}\right|$ is a positive constant re- 


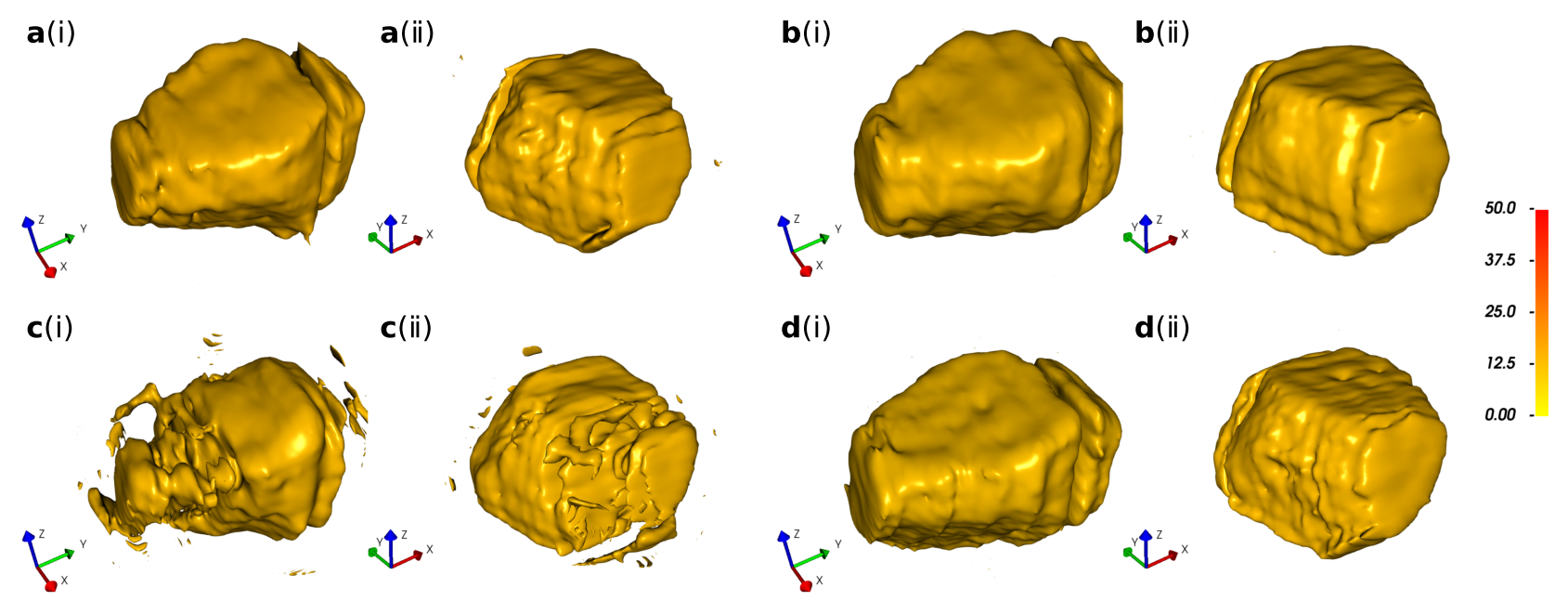

FIG. 2. Amplitude reconstruction of Au nanocrystal. (a,b) Reconstructed amplitude at a $75 \%$ isosurface of the Au nancorystal for the least deformed condition using (a) the HIO algorithm and (b) the Reweighted 2D algorithm. (c,d) Reconstructed amplitude at a $75 \%$ isosurface of the Au nancorystal for the final and most deformed condition using (c) the HIO algorithm and (d) the Reweighted 2D algorithm. It is clearly seen that reconstruction with the HIO algorithm is incomplete and exhibits characteristic drops in amplitude. In contrast, the reweighted $2 \mathrm{D}$ algorithm is able to recover the correct amplitude. (i) and (ii) show alternative projections.

laxation parameter, $\boldsymbol{P}_{\mathrm{M}}=\mathcal{F} \hat{\boldsymbol{P}}_{\mathrm{M}} \mathcal{F}^{-1}$ where $\mathcal{F}$ is the Fourier transform and $n$ is the current iteration.

The rationale here is to eliminate solutions that can be attributed to propagated wavefields of the true object with inherent spatial variations in amplitude that are strongly dependent on propagation distance. At the object plane, these variations are minimal. Application of a weighting factor to the reciprocal-space modulus constraint was found to greatly improve stability in the reconstruction process. To define the iteration step $\Delta \rho^{(n)}$, rather than moving by a fixed one-dimensional proportion in the steepest-descent (steepest-ascent) direction for points within (outside) the support region respectively, each direction is ascribed one of two coupled multiplication factors $\boldsymbol{\tau}^{(n)}=\left(\begin{array}{l}\alpha \\ \beta\end{array}\right)$ that are optimised to orthogonalise successive steps (See Appendix):

$$
\Delta \rho^{(n)}=-\frac{1}{2} \alpha \boldsymbol{P}_{\mathrm{S}} \nabla_{\rho} \mathcal{L}\left(\rho^{(n)}\right)+\frac{1}{2} \beta \boldsymbol{P}_{\underline{\mathrm{S}}} \nabla_{\rho} \mathcal{L}\left(\rho^{(n)}\right)
$$

where $\mathcal{L}(\rho)=\varepsilon_{\mathrm{M}}^{2}(\rho)-\varepsilon_{\mathrm{S}}^{2}(\rho)$ is the error metric difference. Optimisation of $\boldsymbol{\tau}^{(n)}$ proceeds iteratively to establish orthogonality between $\rho^{(n+1)}$ and $\rho^{(n)}+\Delta \rho^{(n)}$. If however $\alpha=1$ and $\beta=$ const., equation 2 reduces to the standard HIO algorithm.

The reweighted two-dimensional (2D) phase retrieval algorithm begins with a calculation of the initial step length $\Delta \rho^{(0)}$ according to equation 2 where $\alpha=1$ and $\beta=0.9$. The gradient of the error metric difference $\nabla_{\rho} \mathcal{L}\left(\rho^{(0)}\right)$ in equation 2 is initially calculated assuming $\boldsymbol{\tau}=\mathbf{0}$. The initial step is used to calculate the next iteration with $\rho^{(1)}=\rho^{(0)}+\Delta \rho^{(0)}$. For subsequent iterations $n, \boldsymbol{\tau}^{(n)}=\boldsymbol{\tau}^{(n-1)}+\boldsymbol{\Delta} \boldsymbol{\tau}^{(n-1)}$ where $\boldsymbol{\Delta} \boldsymbol{\tau}=-\mathcal{H}^{-1} \boldsymbol{\nabla}_{\boldsymbol{\tau}} \psi(\boldsymbol{\tau})$ (equation S10), $\mathcal{H}$ is the Hessian matrix of $\psi(\boldsymbol{\tau})$ and $\psi(\boldsymbol{\tau})=\mathcal{L}(\rho+\Delta \rho)$ (equation
S5). The Hessian matrix $\mathcal{H}$ is then updated according to the Symmetric Rank 1 (SR1) quasi-Newton method (See equation A.11). We then define the following metric:

$$
\varepsilon_{\tau}=\left\|\nabla_{\boldsymbol{\tau}} \psi(\boldsymbol{\tau})\right\|_{2}^{2}
$$

as a measure of orthogonality between $\rho^{(n)}$ and $\rho^{(n)}+$ $\Delta \rho^{(n)}$ (see Equation A.7). If $\varepsilon_{\tau}$ is above a prescribed threshold, $\boldsymbol{\tau}^{(n)}$ is used to recompute $\boldsymbol{\Delta} \boldsymbol{\tau}^{(n-1)}$ according to equation S10. The process of updating the Hessian matrix and recomputing $\boldsymbol{\Delta} \boldsymbol{\tau}^{(n-1)}$ is repeated until $\varepsilon_{\tau}$ falls below the prescribed threshold at which point $\boldsymbol{\Delta} \boldsymbol{\tau}^{(n-1)}$, is kept and used with $\boldsymbol{\tau}^{(n-1)}$ to compute $\Delta \rho^{(n)}$ and in turn $\rho^{(n+1)}$.

\section{B. Sample preparation}

$\mathrm{Au}$ nanocrystals were synthesised via thermal dewetting of a thin Au film. Clean flexible silicon substrates 30 microns in thickness were annealed in $\mathrm{O}_{2}$ at 900 degrees Celsius for 13 hours to grow an oxide layer of $100 \mathrm{~nm}$. Ten nanometres of Au was then deposited using sputter deposition at room temperature and subsequently annealed at 800 degrees Celsius for 12 hours during which dewetting of the Au film resulted in the growth and formation of $\mathrm{Au}$ nanocrystals with well faceted crystalline morphology. Au nanocrystals with dimensions ranging from $100 \mathrm{~nm}$ upto $1 \mu \mathrm{m}$ were observed using scanning electron microscopy. 
a(i)

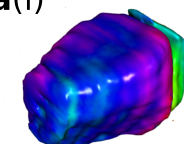

a(ii)

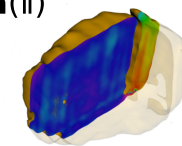

$\mathbf{a}$ (iii)

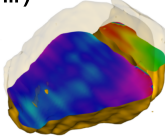

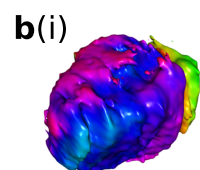

b(ii)

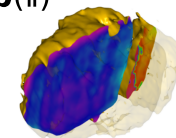

b(iii)

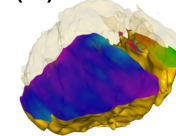

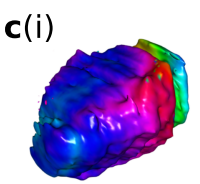

c(ii)

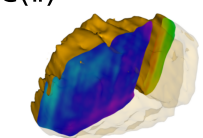

c(iii)

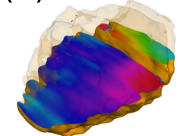

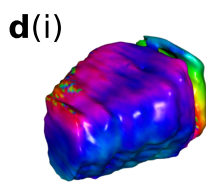

d(ii)

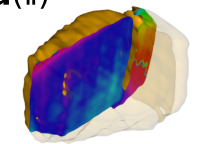

d(iii)

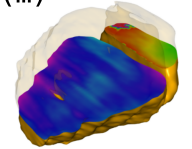

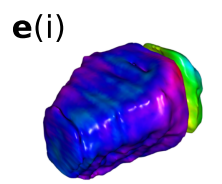

e(ii)

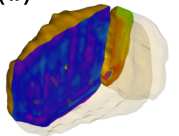

e(iii)

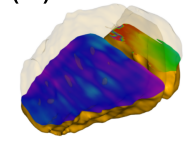

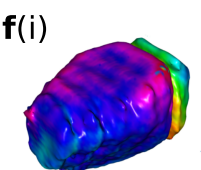

f(ii)

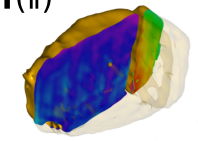

f(iii)

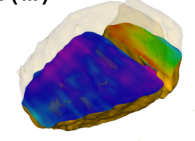

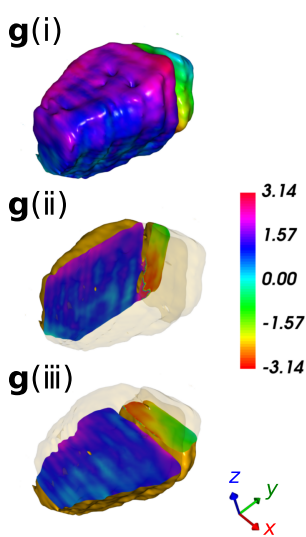

FIG. 3. Reconstructed phase information with increasing deformation. (a(i) - g(i)) Reconstructed amplitude at a $75 \%$ isosurface with phase information mapped onto the surface of the Au nancorystal. (a(ii) - $\mathbf{g}(\mathrm{ii})$ ) Slice through the Au nanocrystal normal to the $x$-axis direction showing phase information. (a(iii) - $\mathbf{g}$ (iii)) Slice through the Au nanocrystal normal to the $z$-axis direction showing phase information. Deformation of the nanocrystal increases from (a) to (g). Increased tensile strain accompanied by phase ripples are observed with increased deformation of the nanocrystal.

\section{BCXDI experimental procedure}

To demonstrate the efficacy of the Reweighted 2D phase retrieval method for the recovery of phase information from a deformed crystalline object, a BCXDI experiment was carried out at beamline I13-1 of the Diamond Light Source synchrotron facility.[30] $\mathrm{Au}$ nanocrystals a few hundred nanometres in size were synthesised on a clean flexible silicon substrate $30 \mathrm{mi}-$ crons thick. The sample was subsequently mounted on a deflection stage that permitted incremental flexing of the substrate by measured amounts while maintaining a fixed axis along the centre line (See figure 1). The substrate was deflected by an initial amount of $10 \mu \mathrm{m}$ (equivalent to a strain within the substrate of $3.75 \times 10^{-7}$ ) to ensure stable mounting during subsequent measurements.

The x-ray beam was monochromated with a paired quartz crystal monochromator and focussed with a Fresnel zone-plate. An order sorting aperture was used to discard higher orders of the zone-plate. Diffraction data was recorded at a distance of 2.5 meters using a Medipix silicon x-ray photon counting detector with a pixel size of $55 \mathrm{\mu m}^{2}$. The $\{111\}$ Bragg reflection of a single $\mathrm{Au}$ nanocrystal, positioned at the fixed central axis, was then illuminated with $9.0 \mathrm{keV}$ x-rays and a speckle pattern recorded. The third dimension of the diffraction

TABLE I. Deformation increments of flexible substrate.

\begin{tabular}{cc|cc}
\hline \hline Increment & Strain $\left(\times 10^{-7}\right)$ & Increment & Strain $\left(\times 10^{-7}\right)$ \\
\hline 1 & 3.750 & 5 & 5.250 \\
2 & 4.125 & 6 & 5.625 \\
3 & 4.500 & 7 & 6.000 \\
4 & 4.875 & & \\
\hline \hline
\end{tabular}

pattern was obtained by the rocking curve method with an increment of 0.005 degrees to satisfy oversampling requirements. Each frame was exposed for 60 seconds.

Incremental deformation of the silicon substrate and hence the Au nanocrystal by a series fixed amounts was subsequently enacted while three-dimensional diffraction data was recorded at each interval. Each tensile strain increment is listed in table I and ranges from $3.750 \times 10^{-7}$ to $6.000 \times 10^{-7}$. Figure $1 \mathrm{~b}$ shows a series of line scans of each recorded speckle pattern showing characteristic asymmetry in the fringe visibility that is attributed to the presence of strain (See also figure S1).

\section{RESULTS AND DISCUSSIONS}

Phase reconstruction was performed by using both the hybrid input-output (HIO) algorithm and the reweighted $2 \mathrm{D}$ phase retrieval method for comparison. Inclusion of other algorithms such as error reduction either preceding or after HIO was found to have no appreciable impact on the outcome. Ten reconstructions were performed for each increment of the mechanical deformation of the Au nanocrystal. Each reconstruction commenced with random phase information with uniform amplitude and was allowed to continue for 10,000 iterations. For the HIO algorithm $\beta=0.9$. For each reconstruction, the support was updated every 50 iterations using the shrink-wrap method. For the reweighted 2D phase retrieval method, $\boldsymbol{\tau}$ was updated with upto 20 iterations of the Symmetric Rank 1 (SR1) quasi-Newton method for each iteration of the algorithm. As reconstruction was performed in the reciprocal-space coordinate system, the final result was geometrically corrected to yield the object in the direct-space rectilinear coordinate system.

The inset of figure 1a shows a scanning electron micrograph of the as synthesised Au nanocrystals which 


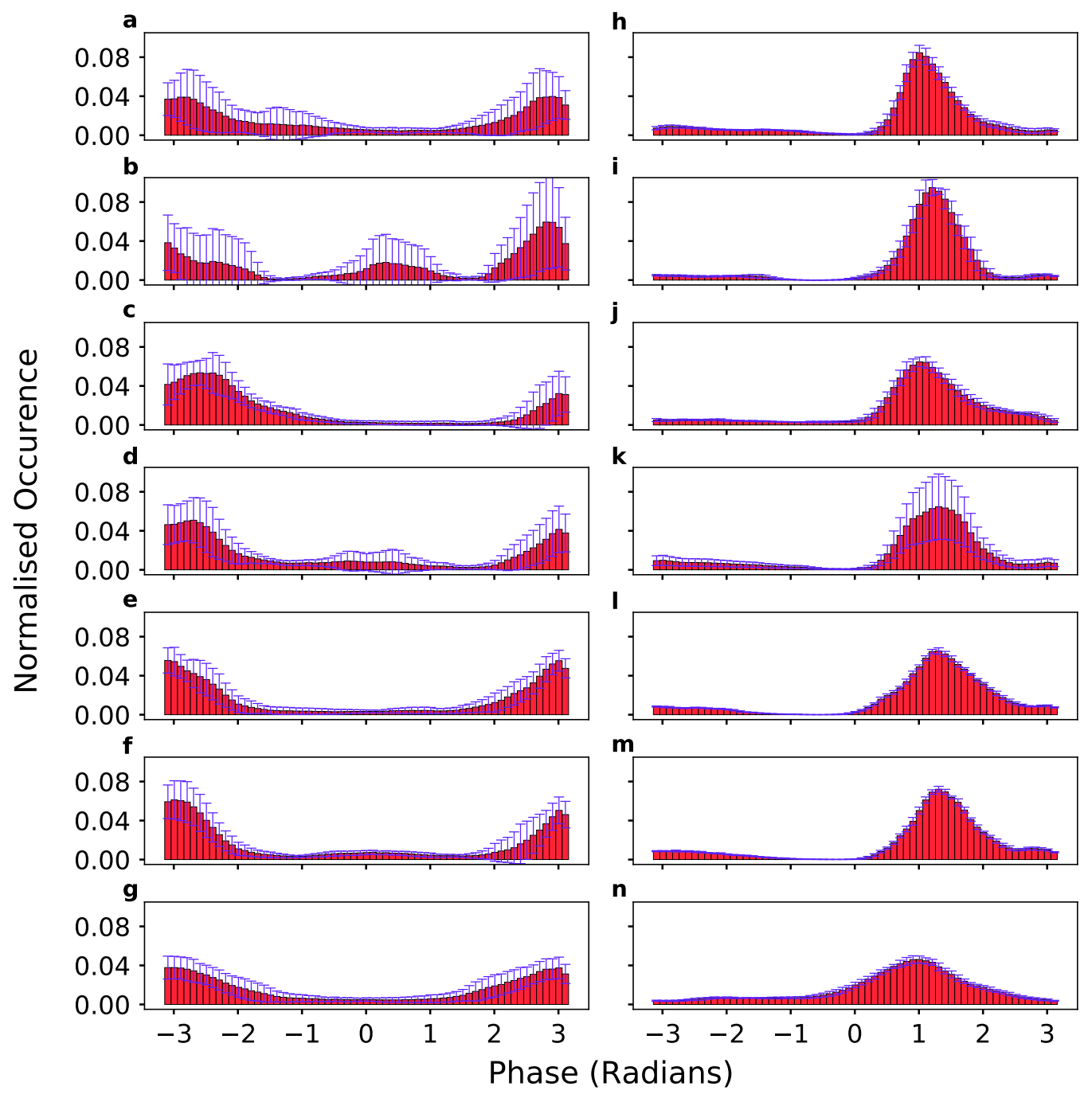

FIG. 4. Normalised histogram of phase information. (a - g) Phase distribution for the Au nanocrystal reconstructed with ten attempts of the HIO algorithm. Deformation is increasing from a to $\mathbf{g}$. Error bars indicate the standard deviation which is indicative of lack of reproducibility in the phase information. (h - $\mathbf{n})$ Phase distribution for the Au nanocrystal reconstructed with ten attempts of the reweighted 2D phase retrieval algorithm. Deformation is increasing from $\mathbf{h}$ to $\mathbf{n}$. Phase information is largely positive in value indicating the presence of tensile strain. Error bars are appreciably narrow indicating good reproducibility in the phase information. The distribution broadens with increasing deformation but remains centred on a positive phase value implying that there is a general increase in positive phase or tensile strain.

appear with faceted morphology due to the equilibrium structure acquired during the thermal annealing process. Figure 2 shows the reconstructed amplitude of the single $\mathrm{Au}$ nanocrystal at an isosurface of $75 \%$ for the least deformed $(\mathbf{a}, \mathbf{b})$ and the most deformed $(\mathbf{c}, \mathbf{d})$ experimental configurations. Figure $2(\mathbf{a}, \mathbf{c})$ shows the amplitude reconstructed with the standard HIO algorithm while $(\mathbf{b}, \mathbf{d})$ are reconstructed with the reweighted $2 \mathrm{D}$ phase retrieval algorithm. For the least deformed case, the phase is predominantly within the range of $\pi$, as can be seen in figure $4 \mathbf{h}$. In this case, both algorithms have performed well and were able to reconstruct the complete morphology to sufficiency. In all reconstruction attempts, the reweighted $2 \mathrm{D}$ phase retrieval algorithm recovered the object without missing density due to wave field propagation. This is distinguished from a thin section of missing density that features in the crystal that is likely due to damage or twinning in the local vicinity (See supplementary video). The success rate of $\mathrm{HIO}$ algorithm was significantly lower at $20 \%$. Crystallographic $\{111\}$ facets are clearly visible and the size of the nanocrystal is consistent with a nominal length of 492 nanometres in each instance. The spatial resolution of each reconstruction was $15 \mathrm{~nm}$.

For the most deformed case, the phase range is beyond $\pi$ as is seen in figure $4 \mathbf{n}$ (discussed below). In this case, the HIO algorithm fails to completely reconstruct the amplitude and exhibits characteristic periodic drops in amplitude (discussed above) that are known to occur when the total phase range is beyond $\pi$. Sim- 
ilar effects are observed for intermediate deformation conditions. The reweighted 2D reconstruction method however is able to reconstruct the complete amplitude of the object while maintaining morphological features found in the reconstructed amplitude for the least deformed condition. In addition, the reweighted $2 \mathrm{D}$ reconstruction method was found to yield narrow amplitude distributions when contrasted with the HIO algorithm as shown in figure S2 (supplementary information) suggesting that the solution obtained is closer to the unpropagated object.[31]
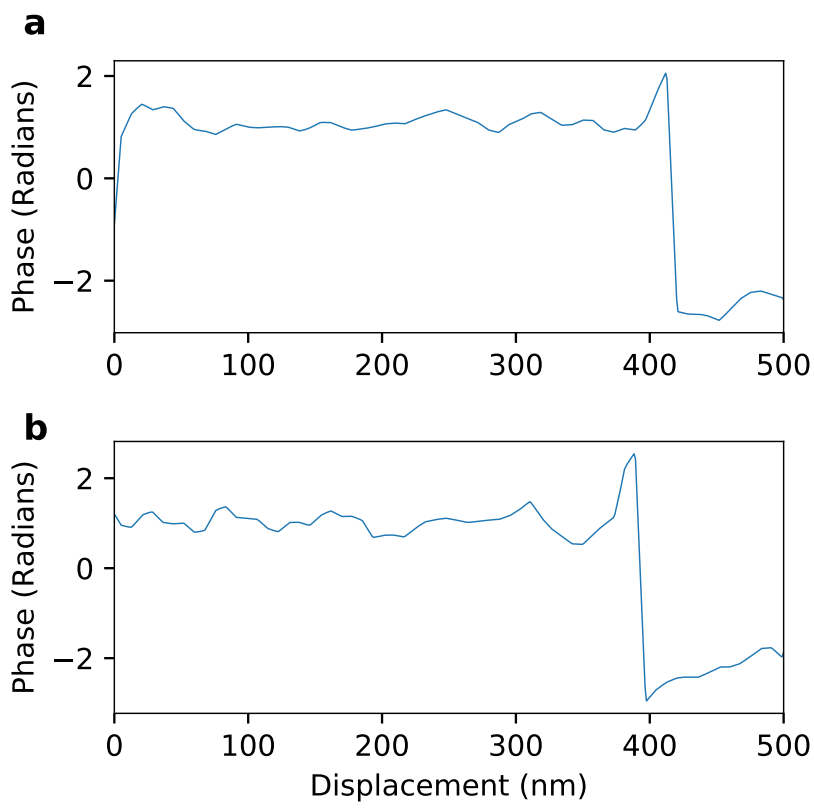

FIG. 5. Phase line scans. a, Phase line scan along the centre line in the $y$-axis ( $Q$-vector) direction of the Au nanocrystal for the least deformed condition. $\mathbf{b}$, Phase line scan along the centre line in the $y$-axis ( $Q$-vector) direction of the $\mathrm{Au}$ nanocrystal for the final and most deformed condition. A phase ripple with periodicity of $\sim 65 \mathrm{~nm}$ is observed.

Figure 3 shows the reconstructed amplitude of the single Au nanocrystal at an isosurface of $75 \%$ for all increments of mechanical deformation, increasing from a to $\mathrm{g}$ for the reweighted 2D phase retrieval algorithm. In (i), the phase information is mapped onto the surface of the nanocrystal and clearly shows predominantly positive phase information which is indicative of tensile strain given that phase information $\phi$ is related to the atomic displacement from the ideal position $\mathbf{u}$ by $\phi=\mathbf{Q} \cdot \mathbf{u}$.[7, 32] In (ii) the Au nanocrystal is sliced into two halves normal to the $x$-axis direction to reveal phase information along the plane. In (iii) slicing occurs normal to the $z$-axis direction. Phase information is seen that is increasingly dominated by tensile strain as the mechanical deformation increases. In addition, ripples in the phase information begin to emerge with a period of $65 \mathrm{~nm}$ along the length of the nanocrystal ( $y$ - axis), which is aligned with the $\mathbf{Q}$-vector This is clearly visible in figure 5 . Their origin is consistent with the formation of a regular distribution of dislocations oriented along the $y$-axis direction (normal to the Burgers vector). Individual dislocations were previously observed in BCXDI experiments and necessarily feature a phase pattern with a $2 \pi$ rotation. $[10,11]$ Although such broad phase variations are not observed, it is conceivable that the resolution of our measurements prohibit visualisation of single dislocations. Other defects such as stacking faults due to shear banding could also induce the observed phase patterns. [33, 34]

A trend toward more tensile strain with increasing deformation is readily observed in figure 4 where normalised histograms of the mean phase information are plotted for both the HIO $(\mathbf{a}-\mathbf{g})$ and reweighted 2D phase retrieval algorithms $(\mathbf{h}-\mathbf{n})$. Deformation increases from a to $\mathbf{g}$ and $\mathbf{h}$ to $\mathbf{n}$. For the HIO algorithm, phase information histograms show no appreciable trend with increasing deformation. Moreover, the standard error is comparable to the occurrence rate which implies that there is significant variation in phase between each reconstruction attempt. For the reweighted 2D phase retrieval algorithm, phase information histograms show predominantly positive phase with a narrow standard error. With increasing deformation, the phase distribution remains largely centred on 1.2 radians while broadening around this point indicates that more regions within the nanocrystal are experiencing tensile strain.

\section{CONCLUSION}

In summary, the use of the reweighted 2D phase retrieval algorithm to recover phase information in BCXDI experiments where the phase is know to extend beyond a range of $\pi$ was demonstrated. The phase reconstruction method demonstrated herein is generally applicable to CDI including both materials science and biological studies. The adoption of reweighted 2D phase retrieval for BCXDI experiments will permit the study of a broad range of materials where phenomena of interest are coupled to lattice distortions. In addition, when conducting ultra-fast stroboscopic BCXDI measurements, as performed at an x-ray free electron laser (XFEL) facility, our findings can enable investigation of dynamic time varying phenomena such as structural phase transitions with high resolution in threedimensions where large lattice distortions are pervasive throughout the material structure and are often prohibitively challenging to reconstruct. Such findings will inform theoretical models and computational simulations of the behaviour of prototypical nanoscale materials during a transition state and will serve as a platform for the development of next generation materials and devices. 


\section{ACKNOWLEDGMENTS}

The authors would like to acknowledge Diamond Light Source for time on beamline I13-1 under proposal number MT10117. M.C.N. conceived and designed the experiments. Experimental data was acquired by M.C.N., X.S. and U.W. Data analysis was performed by M.C.N. The manuscript was prepared by M.C.N. with input from all coauthors.

\section{Appendix: Reweighted Two-Dimensional Phase Retrieval Algorithm}

When coherent diffractive imaging (CDI) measurements are performed phase information is lost and instead we obtain only the reciprocal space scattering intensity distribution $I(\mathbf{q})$ in the form of an interference pattern. This is closely approximated by the square of the modulus of the Fourier transform of the object function $\hat{\rho}(\mathbf{q})$ such that: $I(\mathbf{q})=|\hat{\rho}(\mathbf{q})|^{2}=\left|\hat{\rho}(\mathbf{q}) e^{i \phi(\mathbf{q})}\right|^{2}$. Provided the diffraction pattern is sufficiently oversampled relative to the Nyquist frequency it is possible to recovery the spatially dependent phase shift information $\phi(\mathbf{r})$ and subsequently the electron density map of the object $\rho(\mathbf{r})=|\rho(\mathbf{r})| e^{i \phi(\mathbf{r})}$.

The complex real-space density $\rho(\mathbf{r})$ is recovered using iterative projections between real-space and reciprocalspace while applying a constraint at each turn. The reciprocal-space modulus constraint, $\hat{\boldsymbol{P}}_{\mathrm{M}}$, requires the objects amplitude to be proportional in some way to the original measurement on some set $\mathcal{M}$ such that $\hat{\boldsymbol{P}}_{\mathrm{M}}|\hat{\rho}(\mathbf{q})| e^{i \phi(\mathbf{q})}=\left|\hat{\rho}_{0}(\mathbf{q})\right| e^{i \phi(\mathbf{q})}$, for all $\mathbf{q}$ in $\mathcal{M}$. In addition to the reciprocal-space constraint, defining a support region in real-space where the object's density is unrestricted was found to aid in convergence of the reconstruction process. The real-space support constraint $\boldsymbol{P}_{\mathrm{S}}$ when applied to the density $\rho(\mathbf{r})$ sets all points to zero which are outside of some region $\mathcal{S}$, i.e.: $\mathbf{r} \notin \mathcal{S}$, while the remaining points are unchanged.

Two-dimensional iterative projection phase retrieval is constructed by considering error metrics for the aforementioned modulus and support constraints after $n$ iterations:

$$
\begin{aligned}
\varepsilon_{\mathrm{M}}^{2}(\rho) & =\left\|W^{(n)}(\mathbf{r}) T_{\mathrm{M}}^{(n)}(\mathbf{r})\right\|_{2}^{2} \\
T_{\mathrm{M}}^{(n)} & =\boldsymbol{P}_{\mathrm{M}} \rho(\mathbf{r})^{(n)}-\rho(\mathbf{r})^{(n)} \\
W^{(n)}(\mathbf{r}) & =\left(\left|\rho(\mathbf{r})^{(n-1)}\right|+\epsilon_{0}\right)^{-1} \\
\varepsilon_{\mathrm{S}}^{2}(\rho) & =\left\|T_{\mathrm{S}}^{(n)}(\mathbf{r})\right\|_{2}^{2} \\
T_{\mathrm{S}}^{(n)} & =\boldsymbol{P}_{\mathrm{S}} \rho(\mathbf{r})^{(n)}-\rho(\mathbf{r})^{(n)}
\end{aligned}
$$

where $W(\mathbf{r})$ is a weighting factor that was found to greatly improve stability in the reconstruction process,
$\boldsymbol{P}_{\mathrm{M}}=\mathcal{F} \hat{\boldsymbol{P}}_{\mathrm{M}} \mathcal{F}^{-1}$ where $\mathcal{F}$ is the Fourier transform and $\epsilon_{0} \ll|\rho(\mathbf{r})|$ is a relaxation parameter. The gradients of $\varepsilon_{\mathrm{M}}^{2}$ and $\varepsilon_{\mathrm{S}}^{2}$ are given by:

$$
\begin{gathered}
\nabla_{\rho} \varepsilon_{\mathrm{M}}^{2}=-2 W(\mathbf{r}) T_{\mathrm{M}}(\mathbf{r}), \\
\nabla_{\rho} \varepsilon_{\mathrm{S}}^{2}=-2 T_{\mathrm{S}}
\end{gathered}
$$

from which is can be seen that iterative step lengths of $-\frac{1}{2} \nabla_{\rho} \varepsilon_{\mathrm{M}}^{2}$ and $-\frac{1}{2} \nabla_{\rho} \varepsilon_{\mathrm{S}}^{2}$ will reduce the error metrics to zero. A fixed point of the solution space is then sought by minimising (maximising) the error within (outside) the support region respectively between successive steps:

$$
\min _{\alpha} \max _{\beta} \psi(\alpha, \beta)
$$

Using the error metric difference $\mathcal{L}(\rho)=\varepsilon_{\mathrm{M}}^{2}(\rho)-\varepsilon_{\mathrm{S}}^{2}(\rho)$ yields:

$$
\begin{gathered}
\psi(\alpha, \beta)=\mathcal{L}\left(\rho(\mathbf{r})+\alpha \Delta \rho_{\mathrm{S}}+\beta \Delta \rho_{\underline{\mathrm{S}}}\right), \\
\Delta \rho_{\mathrm{S}}=-\frac{1}{2} \boldsymbol{P}_{\mathrm{S}} \nabla_{\rho} \mathcal{L}(\rho) \\
\Delta \rho_{\underline{\mathrm{S}}}=\frac{1}{2} \boldsymbol{P}_{\underline{\mathrm{S}}} \nabla_{\rho} \mathcal{L}(\rho)
\end{gathered}
$$

where $\boldsymbol{P}_{\underline{\mathrm{S}}}=\boldsymbol{I}-\boldsymbol{P}_{\mathrm{S}}$. Values for $\alpha$ and $\beta$ are sought that bring successive steps of equation A.5 to zero:

$$
\begin{aligned}
& \frac{\partial \psi}{\partial \alpha}=\left\langle-\frac{1}{2} \boldsymbol{P}_{\mathrm{S}} \nabla_{\rho} \mathcal{L}(\rho) \mid \boldsymbol{P}_{\mathrm{S}} \nabla_{\rho} \mathcal{L}\left(\rho(\mathbf{r})+\alpha \Delta \rho_{\mathrm{S}}+\beta \Delta \rho_{\underline{\mathrm{S}}}\right)\right\rangle=0 \\
& \frac{\partial \psi}{\partial \beta}=\left\langle\frac{1}{2} \boldsymbol{P}_{\underline{\mathrm{S}}} \nabla_{\rho} \mathcal{L}(\rho) \mid \boldsymbol{P}_{\underline{\mathrm{S}}} \nabla_{\rho} \mathcal{L}\left(\rho(\mathbf{r})+\alpha \Delta \rho_{\mathrm{S}}+\beta \Delta \rho_{\underline{\mathrm{S}}}\right)\right\rangle=0
\end{aligned}
$$

Equation A.7 can be utilised by rewriting $\alpha$ and $\beta$ as a vector such that:

$$
\begin{aligned}
\boldsymbol{\nabla}_{\boldsymbol{\tau}} \psi(\boldsymbol{\tau}) & =\left\langle\boldsymbol{\Delta} \rho \mid \nabla_{\rho} \mathcal{L}(\rho(\mathbf{r})+\boldsymbol{\tau} \cdot \boldsymbol{\Delta} \rho)\right\rangle=0 \\
\boldsymbol{\tau} & =\left(\begin{array}{c}
\alpha \\
\beta
\end{array}\right) \\
\boldsymbol{\Delta} \rho & =\left(\begin{array}{c}
\Delta \rho_{\mathrm{S}} \\
\Delta \rho_{\underline{\mathrm{S}}}
\end{array}\right) \\
\boldsymbol{\nabla}_{\rho} & =\left(\begin{array}{c}
\boldsymbol{P}_{\mathrm{S}} \nabla_{\rho} \\
\boldsymbol{P}_{\underline{\mathrm{S}}} \nabla_{\rho}
\end{array}\right)
\end{aligned}
$$

where $|\boldsymbol{\tau}| \leq 2.5$. Minimisation of equation A.8 to locate stationary points for $\tau$ can proceed via a number of 
routes with varying degrees of numerical accuracy and speed. An iterative approach to determine $\boldsymbol{\tau}$ utilises the second-order gradient of equation A.8 or equivalently the Hessian $\mathcal{H}$ of $\psi$ :

$$
\mathcal{H}=\left(\begin{array}{cc}
\frac{\partial^{2}}{\partial \alpha^{2}} & \frac{\partial^{2}}{\partial \alpha \partial \beta} \\
\frac{\partial^{2}}{\partial \beta \partial \alpha} & \frac{\partial^{2}}{\partial \beta^{2}}
\end{array}\right) \circ \psi(\boldsymbol{\tau})
$$

which is required to be symmetric and indefinite $\left(\mathcal{H}_{0,0} \geq\right.$ $\left.0, \mathcal{H}_{1,1} \leq 0\right)$. The step length $\boldsymbol{\Delta} \boldsymbol{\tau}$ used to compute the next iteration of $\boldsymbol{\tau}^{(n)}$ is then given by:

$$
\boldsymbol{\Delta} \boldsymbol{\tau}=-\mathcal{H}^{-1} \nabla_{\boldsymbol{\tau}} \psi(\boldsymbol{\tau})
$$

The Hessian matrix is updated iteratively using the Symmetric Rank 1 (SR1) quasi-Newton method, which is a generalisation of the secant root-finding method. It is given by:

$$
\begin{aligned}
\mathcal{H}^{-1} & \rightarrow \mathcal{H}^{-1}+\Delta \mathcal{H}^{-1} \\
\Delta \mathcal{H}^{-1} & =\frac{\left\|\boldsymbol{\Delta} \boldsymbol{\tau}-\mathcal{H}^{-1} \boldsymbol{\nu}\right\|_{2}^{2}}{\left(\boldsymbol{\Delta} \boldsymbol{\tau}-\mathcal{H}^{-1} \boldsymbol{\nu}\right) \cdot \boldsymbol{\nu}} \\
\boldsymbol{\nu} & =\boldsymbol{\nabla}_{\boldsymbol{\tau}} \psi(\boldsymbol{\tau}+\boldsymbol{\Delta} \boldsymbol{\tau})-\boldsymbol{\nabla}_{\boldsymbol{\tau}} \psi(\boldsymbol{\tau})
\end{aligned}
$$

With an initial starting point of $\boldsymbol{\tau}^{(0)}=\mathbf{0}$, by considering equation A.10 and moving in the steepest descentascent direction where $\boldsymbol{\Delta} \boldsymbol{\tau}^{(0)}=\left(\begin{array}{l}1 \\ 1\end{array}\right)$ yields an initial Hessian $\mathcal{H}^{(0)}$ with elements that equate to $-\boldsymbol{\nabla}_{\boldsymbol{\tau}} \psi(\mathbf{0})$ and a unit step in $\tau$. To avoid potential overestimation of feedback in the ascent direction due to nonsmoothness in the error metric difference $\mathcal{L}(\rho)$, it is advantageous to relax feedback in the first iteration so that $\boldsymbol{\tau}^{(1)}=\boldsymbol{\Delta} \boldsymbol{\tau}^{(0)}=\left(\begin{array}{c}1 \\ \beta_{0}\end{array}\right)$, where $\beta_{0} \in[0,1)$. This single relaxation step is equivalent to a single step of the Hybrid Input-Output algorithm. Subsequent steps will iteratively optimise $\boldsymbol{\tau}^{(n)}$ to ensure that successive step directions are orthogonal.
[1] H. N. Chapman and K. A. Nugent, Coherent lensless xray imaging, Nature Photonics 4, 833 EP (2010), review Article.

[2] K. A. Nugent, Coherent methods in the x-ray sciences, Advances in Physics 59, 1 (2010).

[3] J. M. Rodenburg and H. M. L. Faulkner, A phase retrieval algorithm for shifting illumination, Applied Physics Letters 85, 4795 (2004).

[4] J. M. Rodenburg, A. C. Hurst, A. G. Cullis, B. R. Dobson, F. Pfeiffer, O. Bunk, C. David, K. Jefimovs, and I. Johnson, Hard-x-ray lensless imaging of extended objects, Phys. Rev. Lett. 98, 034801 (2007).

[5] J. Miao, T. Ishikawa, I. K. Robinson, and M. M. Murnane, Beyond crystallography: Diffractive imaging using coherent x-ray light sources, Science 348, 530 (2015).

[6] I. Robinson and J. Miao, Three-dimensional coherent x-ray diffraction microscopy, MRS Bulletin 29, 177 (2004).

[7] I. Robinson and R. Harder, Coherent x-ray diffraction imaging of strain at the nanoscale, Nature Materials 8, 291 (2009).

[8] J. N. Clark, L. Beitra, G. Xiong, A. Higginbotham, D. M. Fritz, H. T. Lemke, D. Zhu, M. Chollet, G. J. Williams, M. Messerschmidt, B. Abbey, R. J. Harder, A. M. Korsunsky, J. S. Wark, and I. K. Robinson, Ultrafast three-dimensional imaging of lattice dynamics in individual gold nanocrystals, Science 341, 56 (2013).

[9] M. C. Newton, M. Sao, Y. Fujisawa, R. Onitsuka, T. Kawaguchi, K. Tokuda, T. Sato, T. Togashi, M. Yabashi, T. Ishikawa, T. Ichitsubo, E. Matsubara, Y. Tanaka, and Y. Nishino, Time-resolved coherent diffraction of ultrafast structural dynamics in a single nanowire, Nano Letters 14, 2413 (2014).

[10] J. Ihli, J. N. Clark, A. S. Côté, Y.-Y. Kim, A. S. Schenk,
A. N. Kulak, T. P. Comyn, O. Chammas, R. J. Harder, D. M. Duffy, I. K. Robinson, and F. C. Meldrum, Strainrelief by single dislocation loops in calcite crystals grown on self-assembled monolayers, Nature Communications 7, $11878 \mathrm{EP}$ (2016), article.

[11] J. N. Clark, J. Ihli, A. S. Schenk, Y.-Y. Kim, A. N. Kulak, J. M. Campbell, G. Nisbet, F. C. Meldrum, and I. K. Robinson, Three-dimensional imaging of dislocation propagation during crystal growth and dissolution, Nature Materials 14, 780 EP (2015).

[12] M. C. Newton, S. J. Leake, R. Harder, and I. K. Robinson, Three-dimensional imaging of strain in a single zno nanorod, Nature Materials 9, 120 (2010).

[13] C. Song, D. Ramunno-Johnson, Y. Nishino, Y. Kohmura, T. Ishikawa, C.-C. Chen, T.-K. Lee, and J. Miao, Phase retrieval from exactly oversampled diffraction intensity through deconvolution, Phys. Rev. B 75, 012102 (2007).

[14] R. H. T. Bates, Fourier phase problems are uniquely solvable in more than one dimension i: Underlying theory, Optik 61, 247 (1982).

[15] M. von Laue, Dieäußere Form der Kristalle in ihrem Einfluß auf die Interferenzerscheinungen an Raumgittern., Annalen der Physik 26, 55 (1936).

[16] J. Miao, J. Kirz, and D. Sayre, The oversampling phasing method, Acta Crystallographica Section D 56, 1312 (2000).

[17] M. Pfeifer, G. Williams, I. Vartanyants, R. Harder, and I. Robinson, Three-dimensional mapping of a deformation field inside a nanocrystal, Nature 442, 63 (2006).

[18] T. W. Cornelius, A. Davydok, V. L. R. Jacques, R. Grifone, T. Schülli, M.-I. Richard, G. Beutier, M. Verdier, T. H. Metzger, U. Pietsch, and O. Thomas, In situ three-dimensional reciprocal-space mapping during mechanical deformation, Journal of Synchrotron Radiation 
19, $688(2012)$.

[19] W. Cha, W. Liu, R. Harder, R. Xu, P. H. Fuoss, and S. O. Hruszkewycz, Utilizing broadband X-rays in a Bragg coherent X-ray diffraction imaging experiment, Journal of Synchrotron Radiation 23, 1241 (2016).

[20] W. Cha, A. Ulvestad, M. Allain, V. Chamard, R. Harder, S. J. Leake, J. Maser, P. H. Fuoss, and S. O. Hruszkewycz, Three dimensional variable-wavelength $\mathrm{X}-$ ray bragg coherent diffraction imaging, Phys. Rev. Lett. 117, 225501 (2016).

[21] D. Sayre, Some implications of a theorem due to shannon, Acta Crystallographica 5, 843 (1952).

[22] R. W. Gerchberg and W. O. Saxton, A practical algorithm for the determination of phase from image and diffraction plane apertures, Optik 35, 237 (1972).

[23] J. Fienup, Phase retrieval algorithms - a comparison, Applied Optics 21, 2758 (1982).

[24] X. Huang, R. Harder, G. Xiong, X. Shi, and I. Robinson, Propagation uniqueness in three-dimensional coherent diffractive imaging, Phys. Rev. B 83, 224109 (2011).

[25] X. Shi, J. N. Clark, G. Xiong, X. Huang, R. Harder, and I. K. Robinson, Mechanical breakdown of bent silicon nanowires imaged by coherent x-ray diffraction, New Journal of Physics 15, 123007 (2013).

[26] M. Hayes, The reconstruction of a multidimensional sequence from the phase or magnitude of its fourier transform, IEEE Transactions on Acoustics, Speech, and Signal Processing 30, 140 (1982).
[27] M. C. Newton, R. Harder, X. Huang, G. Xiong, and I. K. Robinson, Phase retrieval of diffraction from highly strained crystals, Phys. Rev. B 82, 165436 (2010).

[28] S. Marchesini, Phase retrieval and saddle-point optimization, J. Opt. Soc. Am. A 24, 3289 (2007).

[29] S. Marchesini, H. He, H. N. Chapman, S. P. Hau-Riege, A. Noy, M. R. Howells, U. Weierstall, and J. C. H. Spence, X-ray image reconstruction from a diffraction pattern alone, Phys. Rev. B 68, 140101 (2003).

[30] C. Rau, U. Wagner, Z. Pešić, and A. De Fanis, Coherent imaging at the diamond beamline i13, physica status solidi (a) 208, 2522 (2011).

[31] See Supplemental Material at [URL will be inserted by publisher] for diffraction pattern images, reconstructed amplitude information and phase reconstruction metrics.

[32] I. Robinson and I. Vartanyants, Use of coherent x-ray diffraction to map strain fields in nanocrystals, Applied Surface Science 182, 186 (2001), proceedings of the International Workshop on Nanomaterials.

[33] P. Li and Z. F. Zhang, Standing wave effect and fractal structure in dislocation evolution, Scientific Reports 7, 4062 (2017).

[34] I. Groma, M. Zaiser, and P. D. Ispánovity, Dislocation patterning in a two-dimensional continuum theory of dislocations, Phys. Rev. B 93, 214110 (2016). 